\title{
Calcium Fluoride Barrier Layer in Tunnel Emitter Phototransistor
}

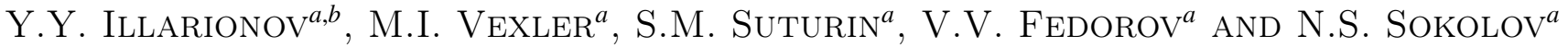 \\ ${ }^{a}$ Ioffe Physical-Technical Institute, 26 Polytechnicheskaya, 194021 St.-Petersburg, Russia \\ ${ }^{b}$ Grenoble Institute of Technology, 46 av. Felix Viallet, 38031 Grenoble, France
}

\begin{abstract}
Owing to the optimized growth technology of the 1-2 nm calcium fluoride films on $n$-(111)-silicon, metal/ tunnel-insulator/semiconductor phototransistors have been fabricated by the molecular beam epitaxy at the temperature $250^{\circ} \mathrm{C}$. The characteristics of these transistors were measured in a wide range of voltages, and the proofs for current gain were found throughout the investigated range. The gain value exceeds $10^{3}$ approaching the theoretically estimated value in this system. The stability and reproducibility of the device characteristics were satisfactory. The results support the candidacy of calcium fluoride for being a vital dielectric in silicon-based functional electronics.
\end{abstract}

PACS: 73.40.Qv, 73.40.Gk, 73.61.Ng

\section{Introduction}

Calcium fluoride $\left(\mathrm{CaF}_{2}\right)$ is a candidate material to be used in the insulating barrier layers in silicon electronics. It is characterized by a wide band gap $\left(E_{\mathrm{g}}=\right.$ $12.1 \mathrm{eV}$ ), large band offsets to $\mathrm{Si}$ (the conduction band discontinuity $\chi_{\mathrm{e}}$ is $2.38 \mathrm{eV}$ ) and high dielectric constant $(\varepsilon=8.43)$ [1]. Due to good lattice matching to silicon $\mathrm{CaF}_{2}$ can be coherently grown on $\mathrm{Si}$ by molecular beam epitaxy (MBE) [2]. Some functional devices such as $\mathrm{Si} / \mathrm{CaF}_{2}$ resonant-tunneling diodes have already been reported $[3,4]$.

In this paper we focus on the bipolar metal-insulatorsemiconductor (MIS) tunnel emitter phototransistor [5]. It is a conventional MIS capacitor whose metal gate is treated as an emitter, the Si bulk as a collector and the inversion layer as a base. The operation of this phototransistor (unlike that of any earlier $\mathrm{CaF}_{2}$ device where only electron tunneling was important) relies on the coexistence of the conduction- and valence band current components [6]. The hole current $j_{\text {ph }}$ photogenerated in the $n$-Si substrate under a reverse bias is spent, at least partially, for leakage into the emitter, so that the additional minority carrier supply changes the insulator bias $U$ and the corresponding electron injection current $j_{\mathrm{e}}$, which is usually larger than the hole component $j_{\mathrm{h}}$.

Our aim was to fabricate such samples with calcium fluoride, to record their current-voltage $(I-V)$ curves in dark and under irradiation, to estimate the gain value and to compare the experimental data with the model predictions. Beyond for the device application this may be important for deeper understanding of the transport phenomena in $\mathrm{CaF}_{2}$ and of the properties of fluoride-based MIS structures in general.

\section{Theoretical background}

Up to now there are well-developed models of MIS tunnel structures, including the transistor action (e.g. [5]). In this section we accentuate the points important specifically for the $\mathrm{Au} / \mathrm{CaF}_{2} / n \mathrm{Si}(111)$ system.

First, for the conduction-band tunneling probability $T$, the value averaged over all the states in the $k$-space with the given carrier energy $E$ and its perpendicular component $E_{\perp}$, was taken. Conservation of a large transverse wave vector $k_{\perp}$ of a tunneling electron in the case of $\mathrm{Si}(111)$ orientation is therefore accounted for, retaining the traditional expressions for $j_{\mathrm{e}}$. This is a substantial improvement compared to Refs. [7, 8].

Secondly, along with $j_{\mathrm{e}}$, the metal-valence band current is treated despite its smallness. Evidently, without $j_{\mathrm{h}}$, transistor action would be impossible as the insulator voltage could not be affected by irradiation. Because of a huge band gap of the fluoride, the tunneling of holes - like of electrons - occurs via the upper barrier (effective mass in $\mathrm{CaF}_{2}$ is $m_{\mathrm{e}}=1.0 m_{0}$ ). As shown in [5], for "one-band" tunneling, non-monotonous behavior of $j_{\mathrm{h}}$ as a function of $U$ at a given terminal bias $V$ is expected. However even with a small addition $j_{\mathrm{h}}=j_{\mathrm{h} \text {,tunn }}+\alpha U_{\mathrm{BE}}$, where $U_{\mathrm{BE}}$ is the Fermi level difference between the metal and the inversion layer, the dependence $j_{\mathrm{h}}(|U|)$ will increase throughout. It may be assumed that $\alpha$ gradually becomes larger in progress of dielectric degradation.

Third, the voltage partitioning is found within the minority-carrier balance model. The losses of holes due to tunneling $j_{\mathrm{h}}$ must be reimbursed by their photogeneration $j_{\mathrm{ph}}$ supply. The photocurrent is modeled as a quantity proportional to the depletion layer width: $j_{\mathrm{ph}}=K w$. Besides a drift-diffusion current 
$j_{\mathrm{dd}}=j_{\mathrm{dd} 0}\left[\exp \left(\Delta E_{\mathrm{Fpn}} / k_{\mathrm{B}} T\right)-1\right]$ flows in Si due to the bulk-interface Fermi level shift $\Delta E_{\mathrm{Fpn}}$. We did not include the thermal generation current $j_{\text {th }} \sim w[5]$ but afforded to adjust the pre-factor of $j_{\text {dd }}$ instead (adopted $\left.j_{\mathrm{dd} 0}=3.75 \times 10^{-6} \mathrm{~A} / \mathrm{cm}^{2}\right)$.

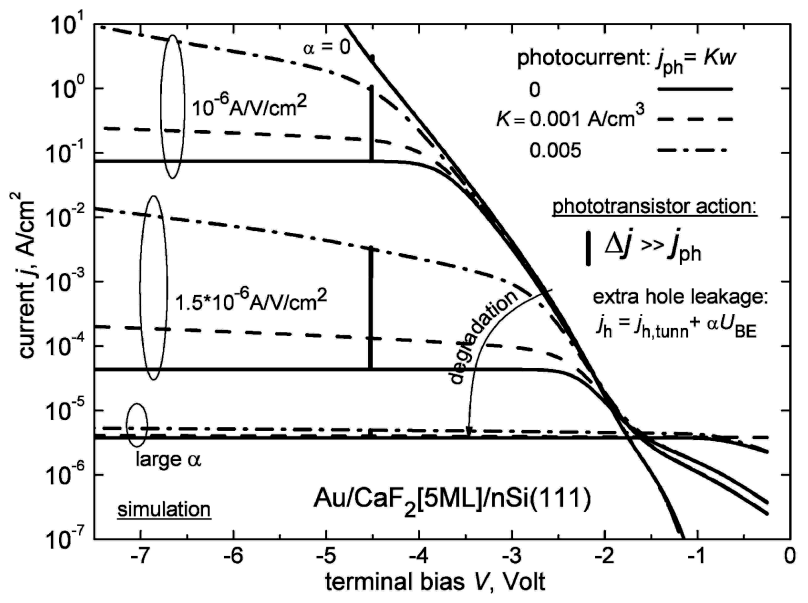

Fig. 1. Simulated $I-V$ curves for the $\mathrm{Au} / \mathrm{CaF}_{2} /$ $n \mathrm{Si}(111)$ system in dark and under irradiation, see text for the details.

The simulation results are shown in Fig. 1. High $|V|$ values are not impractical because the insulator bias $|U|$ in this case is much lower. A nearly exponential increase of the current is seen for $\alpha=0$ (this is saturation, in transistor notation) while for other $\alpha$ the saturation ends at the plateau (active regime). There is an apparent photosensitivity which becomes weaker during degradation (it is a log-scale). Simultaneously, the current decreases because $|U|$ is reduced with the increasing leakage. For $\alpha=0$ the photoresponse is not zero.

While the expected trends are clearly seen in this figure, the gain estimation may be too speculative, particularly because of the $\alpha$ factor. Nevertheless the idea of how high the gain $\beta$ might be, is given by the $j_{\mathrm{e}} / j_{\mathrm{h}}$ component ratio numerically evaluated for a pure tunnel mechanism $\left(j_{\mathrm{h}}=j_{\mathrm{h} \text {,tunn }}\right)$. For example, such evaluations at $|U|=1 \mathrm{~V}$ yield $\beta \approx 65$ for the 3 monolayers ( $3 \mathrm{ML}), \beta \approx 800$ for the $5 \mathrm{ML}$ and $\beta \approx 10^{4}$ for the $7 \mathrm{ML}$ fluoride structures. Let us note that this ratio becomes remarkably larger with the enhancement of the insulator voltage $|U|$, e.g. $\approx 900$ at $1.5 \mathrm{~V}$ for the $3 \mathrm{ML} \mathrm{CaF}_{2}$, mainly due to the rapid increase of the $j_{\mathrm{e}}$ component.

Further we perform the experimental estimation of the gain value.

\section{Sample fabrication}

The $\mathrm{Au} / \mathrm{CaF}_{2} / n \mathrm{Si}(111)$ MIS structures were fabricated by MBE on $n$-type silicon wafers (donor concentration $\left.N_{\mathrm{D}}=10^{15} \mathrm{~cm}^{-3}\right)$. After the standard chemical cleaning procedures [9], the wafers were placed into the MBE chamber. The thermal flash at $1200^{\circ} \mathrm{C}$ was made, and a $7 \times 7 \mathrm{Si}(111)$ superstructure was observed. A several ML thick $(1 \mathrm{ML}=0.315 \mathrm{~nm}) \mathrm{CaF}_{2}$ film was grown at $250^{\circ} \mathrm{C}$ with the rate of $1.5-2 \mathrm{~nm} / \mathrm{min}$. The low-temperature growth allowed for excluding the formation of triangle pinholes in the $\mathrm{CaF}_{2}$ layer whose presence would have made the samples inappropriate for electrical measurements because the local tunnel current density exponentially increases with the decreasing thickness. At the same time, even at $250^{\circ} \mathrm{C}$ the crystalline quality remains satisfactory as confirmed by the reflection high energy electron diffraction. Afterward, gold electrodes (diameter $80 \mu \mathrm{m}$ ) were deposited on top of the grown $\mathrm{CaF}_{2}$ film through the mask. The reason for using gold was that it belongs to the traditional materials for silicon electronics, plus that the $\mathrm{Au} / \mathrm{CaF}_{2}$ barrier height is relatively exactly known $(2.63 \mathrm{eV})$ and stable.

The microscopy studies of the film topography elucidated a very good uniformity. The standard height deviation was less than $0.1 \mathrm{~nm}$ which justifies a disregard for any thickness fluctuations in simulation.

\section{Measurement technique}

In order to prevent a mechanical damage of the $\mathrm{Au}$ electrodes the measurements of the static $I-V$ curves were performed using the atomic force microscope (AFM) with a conductive cantilever in a contact mode which was connected to the electrical scheme.

The voltage generator and ammeter were operated by the program developed in the $\mathrm{C}++$ Builder environment. It enabled to input the measurement parameters of which the integration time, i.e. the time of the current averaging at each fixed voltage $V$, may be worth commenting to. With a large integration time a good accuracy is attained but at risk of sample overload, while a short time warrants more stability but not so high signal-to-noise ratio. We used two options: $400 \mathrm{~ms}$ (primarily for low $V$ ) and 40-70 ms.

A red laser diode (photon energy of $1.8 \mathrm{eV}$ ) served as a light source. This energy suffices for the band-to-band photoeffect in silicon, and is not too large to overthrow the electrons above the $\mathrm{CaF}_{2}$ conduction band barrier. The laser irradiation power was calibrated in arbitrary units so that the measurements for the proportional intensities $\Phi_{0}$ (photocurrent $\left.j_{\mathrm{ph}}=j_{\mathrm{ph} 0}\right), 2 \Phi_{0}\left(j_{\mathrm{ph}}=2 j_{\mathrm{ph} 0}\right)$ etc. were possible.

The measurements were organized as follows. First the $I-V$ characteristics for the negative gate (emitter) bias were recorded in the dark and under light irradiation; so that the difference $\Delta j=j_{\text {light }}-j_{\text {dark }}$ versus $V$ can be found. Then, a high voltage was applied causing a severe overload of the fluoride layer after which the structure ceased to exhibit gain because $U$ and $j_{\mathrm{e} \text {,dam }}$ were not more affected by irradiation. Then the $I-V$ curves in the dark and under irradiation were measured again, all the currents being substantially lower than in the fresh sample case; for the damaged sample, $\Delta j_{\text {dam }}=j_{\text {light,dam }}-j_{\text {dark,dam }} \sim j_{\text {ph }}$. The key point here is that the irradiation condition was exactly the same for the fresh and damaged cases, as no re-landing of the cantilever was necessary. Having obtained $j_{\mathrm{ph}}$ and $\Delta j$, we 
can evaluate the current gain irrespectively of the regime (active, saturation).

\section{Discussion on the experimental results}

In Fig. 2, the results of measurement on the $\mathrm{CaF}_{2}$ MIS tunnel structures are exemplarily presented. The curves are recorded with the large integration time within a rather narrow voltage range near zero. One can see a super-linear increase of a current $j$ with the voltage $|V|$. Under irradiation all the currents become larger. In the inset, the post-overload situation is shown: the currents are incomparably lower than in the main plot, and the photoresponse is also weak. This response equals to the photocurrent $j_{\mathrm{ph}}$ (additional subscript 0 refers to $\Phi_{0}$ ).

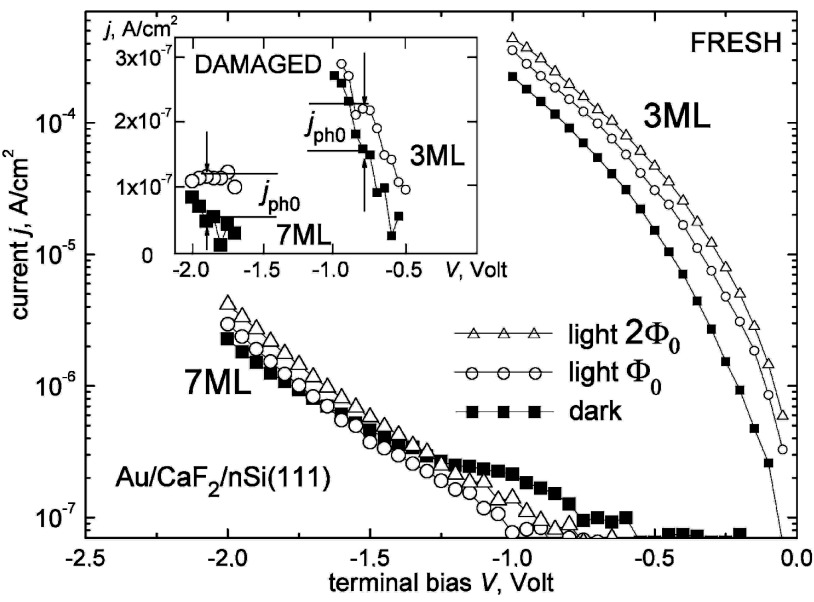

Fig. 2. Experimental characteristics of the fresh (main plot) and damaged (inset) $\mathrm{CaF}_{2}$ MIS tunnel emitter phototransistors in the low-voltage range.

As this photocurrent $j_{\mathrm{ph}}$ is much smaller than the distance $\Delta j$ between the "dark" curve and even the neighbouring curve in the main plot, the phototransistor action may be treated as verified. Such behaviour was observed for all the samples (e.g. [5]). Let us note that for different electrodes the irradiation conditions may be geometrically different, so that the $\Phi_{0}$ value in Fig. 2 is not exactly the same for the $3 \mathrm{ML}$ and $7 \mathrm{ML}$ samples. The current $j_{\mathrm{ph}}$ should therefore be measured for each individual electrode, although the variation is minor.

The current gain $\beta=\left(j_{\text {light }}-j_{\text {dark }}\right) / j_{\text {ph }}-1=\Delta j / j_{\text {ph }}-1 \approx$ $\Delta j / j_{\mathrm{ph}}$, as deduced from these curves, has the order of $10^{2}-10^{3}$. More details on the gain estimation procedure were provided in Ref. [5].

At the attempts to extend the range of $|V|$, sample degradation was encountered: the current gradually decreased qualitatively like in Fig. 1. This made the phototransistor measurements unreliable. However, as mentioned in the previous section, shorter integration time mode allowed for working in much broader voltage ranges. The families of curves obtained for the sample from the same series are represented in Fig. 3 for the range of $[0 \ldots-10] \mathrm{V}$. Let us note that the irradiation intensities used are much higher. As for the dark curves, for the fresh curve they more or less agree to what has been in Fig. 2, but the post-damage currents are evidently higher. This is just a consequence of the accuracy limitation for the short integration times. By the way when the same curves are re-measured with larger integration time, the agreement to Fig. 2 becomes satisfactory.

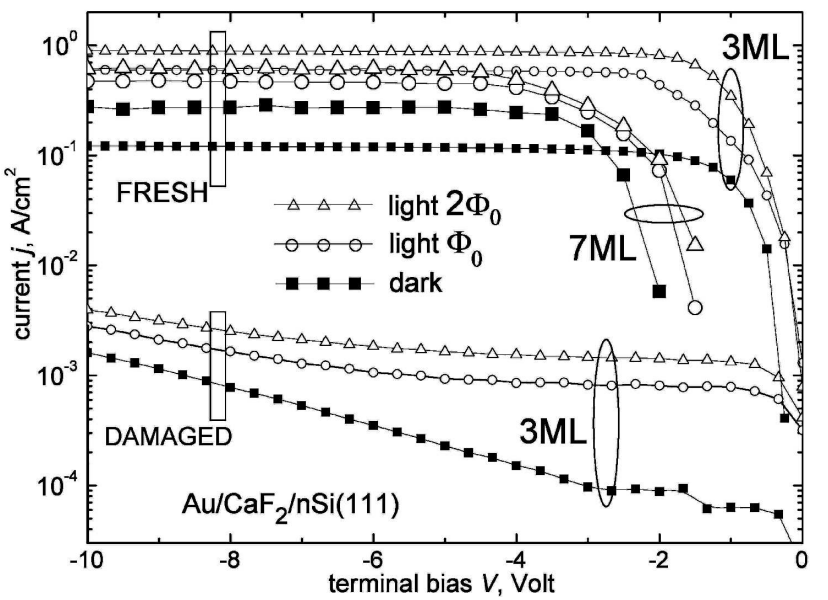

Fig. 3. Experimental characteristics of the $\mathrm{CaF}_{2}$ MIS tunnel emitter phototransistor in the broad voltage range.

In Fig. 3, one can see a rapid current increase with an applied voltage $|V|$ followed by a slowdown, practically up to a plateau, of the current, as usual for MIS tunnel structures in general and as shown in Fig. 1 specifically for the $\mathrm{Au} / \mathrm{CaF}_{2} / n \mathrm{Si}$ structure after its minimal degradation. The reason is simply a deficiency of minority carriers precluding further increase of the insulator voltage $|U|$ with $|V|$ above some limit. Furthermore, an apparent photoresponse is seen, especially in the plateau segments. This concerns both fresh structures and the post-damage case (for which the data are shown just for one sample, to avoid crowding curves). But in the latter situation the reaction to the light was modest. Disposing at these curves the current gain for high voltages can be estimated, like for low voltages, by comparison of $\Delta j$ and $j_{\mathrm{ph}}$. The obtained value of $\beta$ is about $10^{2}$, which is quite good although not larger than in Fig. 2. In general, the measured gain for the $3 \mathrm{ML}$ samples is around the tunnel $j_{\mathrm{e}} / j_{\mathrm{h}}$ ratio values listed at the end of the theoretical section, but for the $7 \mathrm{ML}$ samples it is smaller. It can be admitted that the plateau appears only after some sample degradation. For large integration time, the degradation was too severe to reasonably measure anything afterwards, but with shorter degradation time the recording of the curves is still possible and, in the spirit of what is shown in Fig. 1, we observe clear amplification in the active phototransistor mode. 


\section{Conclusion}

Reverse-bias operation of the $\mathrm{Au} / \mathrm{CaF}_{2} / n \mathrm{Si}(111)$ structures in a wide voltage range revealed their phototransistor properties under external light irradiation. Both saturation and active operation modes were observed, qualitatively typical for the transistors of this type. The current gain was experimentally estimated. It reaches $10^{2}-10^{3}$ which is somewhat lower than the electron-to-hole tunnel component ratio, but still large enough. This result supports the candidacy of calcium fluoride for being a vital dielectric in silicon-based functional electronics.

\section{Acknowledgments}

The authors thank Prof. I.V. Grekhov and Dr. S.E. Tyaginov for stimulating discussion. The support of European Commission via project ONDA FP7-PEOPLE-2009-IRSES-247518 is appreciated.

\section{References}

[1] W. Hayes, Crystals with the Fluorite Structure: Electronic, Vibrational, and Defect Properties, Clarendon, Oxford 1974.

[2] M. Sugiyama, M. Oshima, Microelectron. J. 27, 361 (1996).

[3] M. Watanabe, Y. Iketani, M. Asada, Jpn. J. Appl. Phys. 39, L964 (2000).

[4] S. Watanabe, M. Maeda, T. Sugisaki, K. Tsutsui, Jpn. J. Appl. Phys. 44, 2637 (2005).

[5] M.I. Vexler, Yu.Yu. Illarionov, S.M. Suturin, V.V. Fedorov, N.S. Sokolov, Semicond. Sci. Technol. 25, 095007 (2010).

[6] J.G. Simmons, G.W. Taylor, Solid-State Electron. 29, 287 (1986).

[7] B. Zhang, K. Furuya, Y. Ikeda, N. Kikegawa, Jpn. J. Appl. Phys. 38, 4887 (1999).

[8] M.I. Vexler, N.S. Sokolov, S.M. Suturin, A.G. Banshchikov, S.E. Tyaginov, T. Grasser, J. Appl. Phys. 105, 083716 (2009).

[9] A. Ishizaka, Y. Shiraki, J. Electrochem. Soc. 133, 666 (1986). 